\title{
EXISTENCE RESULTS FOR A BOUNDARY VALUE PROBLEM INVOLVING A FOURTH-ORDER ELASTIC BEAM EQUATION
}

\author{
SHAPOUR HEIDARKHANI*, FARIBA GHAREHGAZLOUEI \\ Department of Mathematics, Faculty of Sciences, Razi University, 67149 Kermanshah, Iran
}

\begin{abstract}
Fourth-order two-point boundary value problems are useful for material mechanics since they usually characterize the deflection of an elastic beam. In this paper, we establish the multiplicity results for a fourth-order differential equation involving Lipschitz non-linearity which models beams on elastic foundations. The approach is based on variational methods and critical point theory.
\end{abstract}

Keywords. Fourth-order boundary value problem; Three solutions; Critical point; Variational methods.

2010 Mathematics Subject Classification. 34B15, 58E05, 49J40.

\section{INTRODUCTION}

Fourth-order boundary value problems which describe the deformations of an elastic beam in an equilibrium state whose both ends are simply supported have been extensively studied in the literature. In this paper, we study the fourth-order boundary value problem

$$
\left\{\begin{array}{l}
u^{(i v)}(x)=\lambda f(x, u(x))+h(u(x)), \quad \text { in }[0,1], \\
u(0)=u^{\prime}(0)=u^{\prime \prime}(1)=0, \\
u^{\prime \prime \prime}(1)=\mu g(u(1)),
\end{array}\right.
$$

where $\lambda>0, \mu \geq 0$ are two parameters. $f:[0,1] \times \mathbb{R} \rightarrow \mathbb{R}$ is an $L^{1}$-Carathéodory function, $g: \mathbb{R} \rightarrow \mathbb{R}$ is a nonnegative continuous function and $h: \mathbb{R} \rightarrow \mathbb{R}$ is a Lipschitz continuous function with the Lipschitz constant $L>0$, i.e.,

$$
\left|h\left(\xi_{1}\right)-h\left(\xi_{2}\right)\right| \leq L\left|\xi_{1}-\xi_{2}\right|, \quad \forall \xi_{1}, \xi_{2} \in \mathbb{R}
$$

such that $h(0)=0$.

Problem (1.1) has the following physical description: a thin flexible elastic beam of length 1 when, along its length, $f$ and $h$ are added to cause deformation. Precisely, condition $u(0)=u^{\prime}(0)=0$ means

${ }^{*}$ Corresponding author.

E-mail addresses: sh.heidarkhani@razi.ac.ir (S. Heidarkhani), f.gharehgazloo@yahoo.com (F. Gharehgazlouei).

Received March 13, 2019; Accepted June 27, 2019.

(C)2019 Journal of Nonlinear Functional Analysis 
that the left end of the beam is fixed and condition $u^{\prime \prime}(1)=0, u^{\prime \prime \prime}(1)=\mu g(u(1))$ means that the right end of the beam is attached to a bearing device, given by the function $g$.

It is well known that fourth-order two-point boundary value problems are useful for material mechanics because the problems usually characterize the deflection of an elastic beam. For this reason, many literatures that deal with the existence results of at least one solution, or multiple solutions, or even infinitely many solutions have been established by many authors using fixed point theorems, lower and upper solutions methods, and critical point theory; see, for example, $[1,2,3,4,5,6,7,8,9,10,11,12$, $13,14,15,16,17]$ and the references therein. These kind of problems arise in real-world phenomena play an important role in different fields of research, such as, mechanical engineering, control systems, economics, computer science, physics, biology and many others. For example, Ma [14] obtained the existence of at least two positive solutions for the following problem

$$
\left\{\begin{array}{l}
u^{(i v)}(x)=f(x, u(x)), \quad \text { in }[0,1], \\
u(0)=u^{\prime}(0)=u^{\prime \prime}(1)=0, \\
u^{\prime \prime \prime}(1)=g(u(1)) .
\end{array}\right.
$$

In [5], Bonanno and Di Bella by using a three critical points theorem (Theorem 2.1) established the existence of at least three weak solutions for the following boundary value problem

$$
\left\{\begin{array}{l}
u^{(i v)}(x)+A u^{\prime \prime}(x)+B u(x)=\lambda f(x, u(x)), \quad \text { in }[0,1], \\
u(0)=u(1)=0, \\
u^{\prime \prime}(0)=u^{\prime \prime}(1)=0,
\end{array}\right.
$$

where $f:[0,1] \times \mathbb{R} \rightarrow \mathbb{R}$ is a function, $A$ and $B$ are real constants and $\lambda$ is a positive parameter. Yang, Chen and Yang [17], by using a variational approach, ensured the existence of three solutions for the problem

$$
\left\{\begin{array}{l}
u^{(i v)}(x)=\lambda f(x, u(x)), \quad \text { in }[0,1] \\
u(0)=u^{\prime}(0)=u^{\prime \prime}(1)=0, \\
u^{\prime \prime \prime}(1)=g(u(1))
\end{array}\right.
$$

Cabada and Tersian [10] established the existence of at least two nontrivial classical solutions for the problem

$$
\left\{\begin{array}{l}
u^{(i v)}(x)+\lambda f(x, u(x))=0, \quad \text { in }[0,1], \\
u(0)=u^{\prime}(0)=u^{\prime \prime}(1)=0, \\
u^{\prime \prime \prime}(1)=\lambda g(u(1)),
\end{array}\right.
$$

where the functions $f:[0,1] \times \mathbb{R} \rightarrow \mathbb{R}$ and $g: \mathbb{R} \rightarrow \mathbb{R}$ are continuous and $\lambda \geq 0$ is a real parameter. Recently, Bonanno, Chinnì and Tersian [4], by using a variational approach, constructed two precise intervals of the parameters $\lambda$ and $\mu$ for which the problem (1.1), in case $h=0$, admits at least one non-zero classical solution ([4] see Theorem 3.1) under conditions involving the antiderivatives of $f$ and g. In [13], the existence of infinitely many generalized solutions for perturbed fourth-order nonlinear boundary value problems with nonlinear boundary conditions, based on variational methods and critical point theory, was investigated. In [12], the authors, based on variational methods and critical point theory, ensured the existence of infinitely many classical solutions the problem (1.1), in the case $h=0$.

In this paper, by using a three critical points theorem (see Theorem 2.1) due Bonanno and Candito established in [18], we discuss the existence of at least three solutions for the problem (1.1), in which two 
parameters are involved. Precise estimates of these two parameters $\lambda$ and $\mu$ will be given. We would like to stress that our results hold under rather different assumptions. In particular, we require that there is a growth of the antiderivative of $f$ which is greater than quadratic in a suitable interval (see, for instance, condition (B1) of Theorem 3.3), and which is less than quadratic in a following suitable interval (see, for instance, condition (B1) of Theorem 3.3). The rest of this paper is arranged as follows. In Section 2, we recall some basic definitions and the main tool (Theorem 2.1). In Section 3, we provide our main result that contains several theorems. Finally, an example is stated to illustrate the results. Precisely, Theorems 3.5 and 3.8 are very special cases of our main results, which are, in fact, Theorem 3.1 and its consequence Theorem 3.3. Theorem 3.5, under suitable conditions on $f$ at zero and at infinity, ensures four distinct non-trivial solutions to the problem (1.1) with $\mu=0$. Theorem 3.8 guarantees three nontrivial solutions to the problem (1.1) with $\lambda=1$.

\section{PRELIMINARIES}

Let

$$
X:=\left\{u \in \mathrm{H}^{2}([0,1]): u(0)=u^{\prime}(0)=0\right\},
$$

where $\mathrm{H}^{2}([0,1])$ is the Sobolev space of all $u:[0,1] \rightarrow \mathbb{R}$ such that $u$ and its distributional derivative $u^{\prime}$ are absolutely continuous and $u^{\prime \prime}$ belongs to $L^{2}([0,1]) . X$ is a Hilbert space with inner product

$$
\langle u, v\rangle:=\int_{0}^{1} u^{\prime \prime}(t) v^{\prime \prime}(t) d t
$$

and norm

$$
\|u\|:=\left(\int_{0}^{1}\left(u^{\prime \prime}(t)\right)^{2} d t\right)^{1 / 2}
$$

which is equivalent to the usual norm $\int_{0}^{1}\left(|u(t)|^{2}+\left|u^{\prime}(t)\right|^{2}+\left|u^{\prime \prime}(t)\right|^{2}\right) d t$. The inclusion $X \hookrightarrow \mathbf{C}^{1}([0,1])$ is compact and it results

$$
\|u\|_{\mathbf{C}^{1}([0,1])}:=\max \left\{\|u\|_{\infty},\left\|u^{\prime}\right\|_{\infty}\right\} \leq\|u\|
$$

for each $u \in \mathbf{X}$. We consider the functionals $\Phi, \Psi_{\lambda, \mu}: X \rightarrow \mathbb{R}$ defined by

$$
\Phi(u):=\frac{1}{2}\|u\|^{2}-\int_{0}^{1} H(u(x)) d x
$$

and

$$
\Psi_{\lambda, \mu}(u):=\int_{0}^{1} F(x, u(x)) d x+\frac{\mu}{\lambda} G(u(1))
$$

for each $u \in X$ and for each $\lambda, \mu>0$, where $F(x, \xi):=\int_{0}^{\xi} f(x, t) d t, G(\xi):=\int_{0}^{\xi} g(t) d t$ and $H(\xi):=$ $\int_{0}^{\xi} h(t) d t$ for each $x \in[0,1], \xi \in \mathbb{R}$.

Suppose that the Lipschitz constant $L>0$ of function $h$ satisfies $L<1$. The key theorem to prove our main result is [18, Theorem 3.3] due to Bonanno and Candito. For the reader's convenience we here recall its immediate consequence (see also [2, Theorem 5.2]).

For all $r, r_{1}, r_{2}>\inf _{X} \Phi, r_{2}>r_{1}, r_{3}>0$, we define

$$
\begin{aligned}
& \varphi(r):=\inf _{u \in \Phi^{-1}(-\infty, r)} \frac{\sup _{u \in \Phi^{-1}(-\infty, r)} \Psi(u)-\Psi(u)}{r-\Phi(u)}, \\
& \beta\left(r_{1}, r_{2}\right):=\inf _{u \in \Phi^{-1}\left(-\infty, r_{1}\right)} \sup _{v \in \Phi^{-1}\left[r_{1}, r_{2}\right)} \frac{\Psi(v)-\Psi(u)}{\Phi(v)-\Phi(u)},
\end{aligned}
$$




$$
\gamma\left(r_{2}, r_{3}\right):=\frac{\sup _{u \in \Phi^{-1}\left(-\infty, r_{2}+r_{3}\right)} \Psi(u)}{r_{3}}
$$

and

$$
\alpha\left(r_{1}, r_{2}, r_{3}\right):=\max \left\{\varphi\left(r_{1}\right), \varphi\left(r_{2}\right), \gamma\left(r_{2}, r_{3}\right)\right\}
$$

Theorem 2.1. Let $X$ be a reflexive real Banach space. Let $\Phi: X \rightarrow \mathbb{R}$ be a convex, coercive and continuously Gâteaux differentiable functional whose Gâteaux derivative admits a continuous inverse on $X^{*}$. Let $\Psi: X \rightarrow \mathbb{R}$ be a continuously Gâteaux differentiable functional whose Gâteaux derivative is compact such that

$\left(\mathrm{a}_{1}\right) \Phi(0)=\Psi(0)=0$.

(a) for every $u_{1}, u_{2}$ such that $\Psi\left(u_{1}\right) \geq 0$ and $\Psi\left(u_{2}\right) \geq 0$ one has

$$
\inf _{t \in[0,1]} \Psi\left(t u_{1}+(1-t) u_{2}\right) \geq 0 .
$$

Assume that there are three positive constants $r_{1}, r_{2}, r_{3}$ with $r_{1}<r_{2}$ such that

(a) $\varphi\left(r_{1}\right)<\beta\left(r_{1}, r_{2}\right)$;

(a) $\varphi\left(r_{2}\right)<\beta\left(r_{1}, r_{2}\right)$;

(a) $\gamma\left(r_{2}, r_{3}\right)<\beta\left(r_{1}, r_{2}\right)$.

Then, for each $\lambda \in] \frac{1}{\beta\left(r_{1}, r_{2}\right)}, \frac{1}{\alpha\left(r_{1}, r_{2}, r_{3}\right)}[$, the functional $\Phi-\lambda \Psi$ admits at least three distinct critical points $u_{1}, u_{2}, u_{3}$ such that $u_{1} \in \Phi^{-1}\left(-\infty, r_{1}\right), u_{2} \in \Phi^{-1}\left[r_{1}, r_{2}\right)$ and $u_{3} \in \Phi^{-1}\left(-\infty, r_{2}+r_{3}\right)$.

We refer the interested reader to the papers [19, 20,21] in which Theorem 2.1 has been successfully employed to obtain the existence of at least three solutions for boundary value problems.

We assume throughout, and without further mention, that the following conditions for the function $f$ hold:

$\left(\mathrm{b}_{1}\right)$ for every $\rho>0$, there exists a function $l_{\rho} \in L^{1}([0,1])$ such that

$$
\sup _{|t| \leq \rho}|f(x, t)| \leq l_{\rho}(x)
$$

for almost every $x \in[0,1]$;

(b $\left.b_{2}\right) f(x, t) t \geq 0$ for almost every $x \in[0,1]$ and for all $t \in \mathbb{R}$.

When the Maximum Principle holds, we can substitute $\left(\mathrm{b}_{2}\right)$ with

$\left(b_{2}^{\prime}\right) f(x, t) \geq 0$ for almost every $x \in[0,1]$ and for all $t \geq 0$. Of course, when $\left(\mathrm{b}_{2}^{\prime}\right)$ holds, maybe $f(x, 0) \neq 0$.

It is easy to see that if $f(x, t) t<0$ for every $x \in[0,1]$ and $t \neq 0$, then problem (1.1) has only the trivial solution.

Let us recall that a weak solution of problem (1.1) is a function $u \in X$ such that

$$
\int_{0}^{1} u^{\prime \prime}(x) v^{\prime \prime}(x) d x-\int_{0}^{1} h(u(x)) v(x) d x-\lambda \int_{0}^{1} f(x, u(x)) v(x) d x-\mu g(u(1)) v(1)=0
$$

for every $v \in X$. Moreover, a function $u:[0,1] \rightarrow \mathbb{R}$ is said to be a generalized solution to problem (1.1) if $u \in C^{3}([0,1]), u^{\prime \prime \prime} \in A C([0,1]), u(0)=u^{\prime}(0)=u^{\prime \prime}(1)=0, u^{\prime \prime \prime}(1)=\mu g(u(1))$, and $u^{(i v)}(x)=\lambda f(x, u(x))+$ $h(u(x))$ for almost every $x \in[0,1]$. If $f$ is continuous in $[0,1] \times \mathbb{R}$, then each generalized solution is a classical solution. 


\section{MAIN RESULTS}

For $\alpha \geq 0$, set

$$
G^{\alpha}:=\max _{|\xi| \leq \alpha} G(\xi)
$$

We formulate our main result as follows.

Theorem 3.1. Assume that there exist four positive constants $c_{1}, d, c_{2}, c_{3}$ with

$$
c_{1}<d<\frac{3 \sqrt{3}}{8 \pi^{2}} \sqrt{\frac{1-L}{1+L}} c_{2}<\frac{3 \sqrt{3}}{8 \pi^{2}} \sqrt{\frac{1-L}{1+L}} c_{3}
$$

such that

$\left(\mathrm{A}_{1}\right) \quad \max \left\{\frac{\int_{0}^{1} F\left(x, c_{1}\right) d x}{c_{1}^{2}}, \frac{\int_{0}^{1} F\left(x, c_{2}\right) d x}{c_{2}^{2}}, \frac{\int_{0}^{1} F\left(x, c_{3}\right) d x}{c_{3}^{2}-c_{2}^{2}}\right\}<\frac{\left(\frac{3}{2}\right)^{3}(1-L)}{1+L} \frac{\int_{\frac{3}{4}}^{1} F(x, d) d x-\int_{0}^{1} F\left(x, c_{1}\right) d x}{8 \pi^{4} d^{2}}$.

Then, for every

$$
\begin{array}{r}
\lambda \in] \frac{\left(\frac{2}{3}\right)^{3}(1+L) 4 \pi^{4} d^{2}}{\int_{\frac{3}{4}}^{1} F(x, d) d x-\int_{0}^{1} F\left(x, c_{1}\right) d x}, \\
\min \left\{\frac{(1-L) c_{1}^{2}}{2 \int_{0}^{1} F\left(x, c_{1}\right) d x}, \frac{(1-L) c_{2}^{2}}{2 \int_{0}^{1} F\left(x, c_{2}\right) d x}, \frac{(1-L)\left(c_{3}^{2}-c_{2}^{2}\right)}{2 \int_{0}^{1} F\left(x, c_{3}\right) d x}\right\}[,
\end{array}
$$

and for each nonnegative continuous function $g: \mathbb{R} \rightarrow \mathbb{R}$, there exists $\delta_{\lambda, g}>0$ given by

$$
\begin{array}{r}
\min \left\{\operatorname { m i n } \left\{\frac{(1-L) c_{1}^{2}-2 \lambda \int_{0}^{1} F\left(x, c_{1}\right) d x}{G^{c_{1}}}, \frac{(1-L) c_{2}^{2}-2 \lambda \int_{0}^{1} F\left(x, c_{2}\right) d x}{G^{c_{2}}},\right.\right. \\
\left.\frac{(1-L)\left(c_{3}^{2}-c_{2}^{2}\right)-2 \lambda \int_{0}^{1} F\left(x, c_{3}\right) d x}{G^{c_{3}}}\right\} \\
\left.\frac{\left(\frac{2}{3}\right)^{3}(1+L) 4 \pi^{4} d^{2}-\lambda\left(\int_{\frac{3}{4}}^{1} F(x, d) d x-\int_{0}^{1} F\left(x, c_{1}\right) d x\right)}{G(d)-G^{c_{1}}}\right\}
\end{array}
$$

such that, for each $\mu \in\left[0, \delta_{\lambda, g}\right.$, problem (1.1) admits at least three generalized solutions $u_{i}(i=1,2,3)$ such that $\left\|u_{i}\right\|_{\infty}<c_{3}, i=1,2,3$.

Proof. Our goal is to apply Theorem 2.1 to problem (1.1). To this end, fix $\lambda$, and nonnegative continuous function $g: \mathbb{R} \rightarrow \mathbb{R}$ and $\mu$ as in the conclusion. For each $u \in X$, let the functionals $\Phi, \Psi_{\lambda, \mu}: X \rightarrow \mathbb{R}$ be defined by

and

$$
\Phi(u):=\frac{1}{2}\|u\|^{2}-\int_{0}^{1} H(u(x)) d x
$$

$$
\Psi_{\lambda, \mu}(u):=\int_{0}^{1} F(x, u(x)) d x+\frac{\mu}{\lambda} G(u(1)) .
$$

Let us prove that the functionals $\Phi$ and $\Psi$ satisfy the required conditions in Theorem 2.1. It is well known that $\Psi_{\lambda, \mu}$ is a differentiable functional whose differential at the point $u \in X$ is

$$
\left\langle\Psi_{\lambda, \mu}^{\prime}(u), v\right\rangle=\int_{0}^{1} f(x, u(x)) v(x) d x+\frac{\mu}{\lambda} g(u(1)) v(1)
$$

for every $v \in X$, and $\Psi_{\lambda, \mu}^{\prime}: X \longrightarrow X^{*}$ is a compact operator. Indeed, it is enough to show that $\Psi_{\lambda, \mu}^{\prime}$ is strongly continuous on $X$. For this end, for fixed $u \in X$, let $u_{n} \rightarrow u$ weakly in $X$ as $n \rightarrow \infty$. Then 
$u_{n}(x)$ converges uniformly to $u(x)$ on $[0,1]$ as $n \rightarrow \infty$; see [22]. Since $f, g$ are continuous in $\mathbb{R}$ for every $x \in[0,1]$. So

$$
f\left(x, u_{n}(x)\right)+\frac{\mu}{\lambda} g\left(u_{n}(1)\right) \rightarrow f(x, u(x))+\frac{\mu}{\lambda} g(u(1)) \text { as } n \rightarrow \infty .
$$

Thus $\Psi_{\lambda, \mu}^{\prime}\left(u_{n}\right) \rightarrow \Psi_{\lambda, \mu}^{\prime}(u)$ as $n \rightarrow \infty$. Hence we proved that $\Psi_{\lambda, \mu}^{\prime}$ is a compact operator by the proposition 26.2 of [22]. Now from the fact $-L|\xi| \leq h(\xi) \leq L|\xi|$ for all $\xi \in \mathbb{R}$, and taking (2.1) into account for every $u \in X$, we have

$$
\frac{1-L}{2}\|u\|^{2} \leq \Phi(u) \leq \frac{1+L}{2}\|u\|^{2}
$$

Clearly, $\Phi$ is a convex and coercive. Furthermore, $\Phi$ is continuously differentiable whose differential at the point $u \in X$ is

$$
\left\langle\Phi^{\prime}(u), v\right\rangle=\int_{0}^{1} u^{\prime \prime}(x) v^{\prime \prime}(x) d x-\int_{0}^{1} h(u(x)) v(x) d x, \quad \forall v \in X .
$$

Also, $\Phi^{\prime}: X \longrightarrow X^{*}$ admits a continuous inverse on $X^{*}$. Indeed, for every $u \in X \backslash\{0\}$,

$$
\begin{aligned}
\lim _{\|u\| \rightarrow \infty} \frac{\left\langle\Phi^{\prime}(u), u\right\rangle}{\|u\|} & =\lim _{\|u\| \rightarrow \infty} \frac{\int_{0}^{1}\left(u^{\prime \prime}(x) u^{\prime \prime}(x)\right) d x-\int_{0}^{1} h(u(x)) u(x) d x}{\|u\|} \\
& \geq \lim _{\|u\| \rightarrow \infty} \frac{(1-L)\|u\|^{2}}{2\|u\|} \\
& =\lim _{\|u\| \rightarrow \infty} \frac{(1-L)\|u\|}{2}=\infty .
\end{aligned}
$$

So, $\Phi^{\prime}(u)$ is coercive. Now, for any $u, v \in X$, one has

$$
\begin{aligned}
\left\langle\Phi^{\prime}(u)-\Phi^{\prime}(v), u-v\right\rangle & =\int_{0}^{1}\left(u^{\prime \prime}(x)-v^{\prime \prime}(x)\right)\left(u^{\prime \prime}(x)-v^{\prime \prime}(x)\right) d x \\
& -\int_{0}^{1}(h(u(x))-h(u(x)))(u(x)-v(x)) d x \\
& \geq \frac{1-L}{2}\|u-v\|^{2} .
\end{aligned}
$$

Thus, $\Phi^{\prime}(u)$ is uniformly monotone. By [22, Theorem 26.A (d)], $\Phi^{\prime-1}$ exists and is continuous on $X^{*}$. Therefore, we observe that the regularity assumptions on $\Phi$ and $\Psi_{\lambda, \mu}$, as requested of Theorem 2.1, are satisfied. Let

$$
\bar{v}(x)= \begin{cases}0 & x \in\left[0, \frac{3}{8}\right], \\ d \cos ^{2}\left(\frac{4 \pi x}{3}\right) & x \in] \frac{3}{8}, \frac{3}{4}[, \\ d & x \in\left[\frac{3}{4}, 1\right] .\end{cases}
$$

Simple calculations show that $\|\bar{v}\|^{2}=8 \pi^{4} d^{2}\left(\frac{2}{3}\right)^{3} \cdot \bar{v} \in X$. It follows from (3.1) that

$$
4 \pi^{4}(1-L) d^{2}\left(\frac{2}{3}\right)^{3} \leq \Phi(\bar{v}) \leq 4 \pi^{4}(1+L) d^{2}\left(\frac{2}{3}\right)^{3} .
$$

Putting $r_{1}=\frac{1-L}{2} c_{1}^{2}, r_{2}=\frac{1-L}{2} c_{2}^{2}$ and $r_{3}=\frac{1-L}{2}\left(c_{3}^{2}-c_{2}^{2}\right)$, the condition

$$
c_{1}<d<\frac{3 \sqrt{3}}{8 \pi^{2}} \sqrt{\frac{1-L}{1+L}} c_{2}<\frac{3 \sqrt{3}}{8 \pi^{2}} \sqrt{\frac{1-L}{1+L}} c_{3}
$$


in conjunction with (3.3) yields $r_{1}<\Phi(\bar{v})<r_{2}$, and also we observe that $r_{3}>0$. Moreover, taking (2.1) into account, one has $\max _{x \in[0,1]}|u(x)| \leq c_{1}$ when $\Phi(u)<r_{1}$. Since $f$ and $g$ are nonnegative, we obtain

$$
\begin{aligned}
\sup _{u \in \Phi^{-1}\left(-\infty, r_{1}\right)} \int_{0}^{1}\left[F(x, u(x)) d x+\frac{\mu}{\lambda} G(u(1))\right] & \leq \int_{0}^{1} \max _{|t| \leq c_{1}} F(x, t) d x+\frac{\mu}{\lambda} G^{c_{1}} \\
& =\int_{0}^{1} F\left(x, c_{1}\right) d x+\frac{\mu}{\lambda} G^{c_{1}} .
\end{aligned}
$$

In a similar way, we get

$$
\sup _{u \in \Phi^{-1}\left(-\infty, r_{2}\right)} \int_{0}^{1}\left[F(x, u(x)) d x+\frac{\mu}{\lambda} G(u(1))\right] \leq \int_{0}^{1} F\left(x, c_{2}\right) d x+\frac{\mu}{\lambda} G^{c_{2}}
$$

and

$$
\sup _{u \in \Phi^{-1}\left(-\infty, r_{2}+r_{3}\right)} \int_{0}^{1}\left[F(x, u(x)) d x+\frac{\mu}{\lambda} G(u(1))\right] \leq \int_{0}^{1} F\left(x, c_{3}\right) d x+\frac{\mu}{\lambda} G^{c_{3}} .
$$

Therefore, since $0 \in \Phi^{-1}\left(-\infty, r_{1}\right)$, one has

$$
\begin{gathered}
\varphi\left(r_{1}\right) \leq \frac{\sup _{u \in \Phi^{-1}\left(-\infty, r_{1}\right)} \Psi(u)}{r_{1}} \leq \frac{\int_{0}^{1} F\left(x, c_{1}\right) d x+\frac{\mu}{\lambda} G^{c_{1}}}{\frac{1-L}{2} c_{1}^{2}} \\
\varphi\left(r_{2}\right) \leq \frac{\sup _{u \in \Phi^{-1}\left(-\infty, r_{2}\right)} \Psi(u)}{r_{2}} \leq \frac{\int_{0}^{1} F\left(x, c_{2}\right) d x+\frac{\mu}{\lambda} G^{c_{2}}}{\frac{1-L}{2} c_{2}^{2}},
\end{gathered}
$$

and

$$
\gamma\left(r_{2}, r_{3}\right) \leq \frac{\sup _{u \in \Phi^{-1}\left(-\infty, r_{2}+r_{3}\right)} \Psi(u)}{r_{3}} \leq \frac{\int_{0}^{1} F\left(x, c_{3}\right) d x+\frac{\mu}{\lambda} G^{c_{3}}}{\frac{1-L}{2}\left(c_{3}^{2}-c_{2}^{2}\right)} .
$$

On the other hand,

$$
\int_{0}^{1} F(x, \bar{v}(x))+\frac{\mu}{\lambda} G(d) \geq \int_{\frac{3}{4}}^{1} F(x, d)+\frac{\mu}{\lambda} G(d) .
$$

For each $u \in \Phi^{-1}\left(-\infty, r_{1}\right)$, one has

$$
\begin{aligned}
\beta\left(r_{1}, r_{2}\right) & \left.\geq \frac{\int_{0}^{1} F(x, \bar{v}(x)) d x+\frac{\mu}{\lambda} G(d)-\int_{0}^{1} F(x, u(x)) d x-\frac{\mu}{\lambda} G(u(1))}{4 \pi^{4}(1+L) d^{2}\left(\frac{2}{3}\right)^{3}-\frac{1-L}{2}\|u\|^{2}}\right) \\
& \geq \frac{\int_{0}^{1} F(x, \bar{v}(x)) d x+\frac{\mu}{\lambda} G(d)-\int_{0}^{1} F\left(x, c_{1}\right) d x-\frac{\mu}{\lambda} G^{c_{1}}}{4 \pi^{4}(1+L) d^{2}\left(\frac{2}{3}\right)^{3}} \\
& \geq \frac{\int_{\frac{3}{4}}^{1} F(x, d) d x+\frac{\mu}{\lambda} G(d)-\int_{0}^{1} F\left(x, c_{1}\right) d x-\frac{\mu}{\lambda} G^{c_{1}}}{4 \pi^{4}(1+L) d^{2}\left(\frac{2}{3}\right)^{3}} .
\end{aligned}
$$

From $\left(A_{1}\right)$, we obtain

$$
\alpha\left(r_{1}, r_{2}, r_{3}\right)<\beta\left(r_{1}, r_{2}\right) .
$$

Finally, we verify that $\Phi-\lambda \Psi_{\lambda, \mu}$ satisfies the assumption $\left(a_{2}\right)$ of Theorem 2.1. Let $u_{1}$ and $u_{2}$ be two local minima for $\Phi-\lambda \Psi_{\lambda, \mu}$. Then $u_{1}$ and $u_{2}$ are critical points for $\Phi-\lambda \Psi_{\lambda, \mu}$, and so, they are weak solutions of problem (1.1). Since $f$ and $g$ are non-negative, for fixed $\lambda>0$ and $\mu \geq 0$, we have 
$(\lambda f+\mu g)\left(x, s u_{1}+(1-s) u_{2}\right) \geq 0$ for all $s \in[0,1]$. Consequently, $\Psi_{\lambda, \mu}\left(s u_{1}+(1-s) u_{2}\right) \geq 0$, for every $s \in[0,1]$. Hence, Theorem 2.1 implies that for every

$$
\begin{array}{r}
\lambda \in] \frac{\left(\frac{2}{3}\right)^{3}(1+L) 4 \pi^{4} d^{2}}{\int_{\frac{3}{4}}^{1} F(x, d) d x-\int_{0}^{1} F\left(x, c_{1}\right) d x}, \\
\min \left\{\frac{(1-L) c_{1}^{2}}{2 \int_{0}^{1} F\left(x, c_{1}\right) d x}, \frac{(1-L) c_{2}^{2}}{2 \int_{0}^{1} F\left(x, c_{2}\right) d x}, \frac{(1-L)\left(c_{3}^{2}-c_{2}^{2}\right)}{2 \int_{0}^{1} F\left(x, c_{3}\right) d x}\right\}[,
\end{array}
$$

and $\mu \in\left[0, \delta_{\lambda, g}\right)$, the functional $I_{\lambda, \mu}$ has three critical points $u_{i}, i=1,2,3$, in $X$ such that $\Phi\left(u_{i}\right)<r_{1}+r_{2}$, that is, $\left\|u_{i}\right\|_{\infty}<c_{3}, i=1,2,3$. Then, taking into account the fact that the weak solutions of problem (1.1) are exactly critical points of the functional $I_{\lambda, \mu}$, which they are also generalized solutions, we have the desired conclusion.

Remark 3.2. We observe that if $f$ and $g$ are positive and continuous, then we obtain three positive classical solutions. Clearly, if we assume condition $\left(b_{2}^{\prime}\right)$ instead of condition $\left(b_{2}\right)$, the solutions ensured by Theorem 3.1 are nontrivial. In the case that $f(x, 0)=0$, we have nontrivial solutions if we assume the following two conditions: Assume that there are a non-empty open set $J \subseteq[0,1]$ and $j \subset J$ of positive Lebesgue measure such that

$\left(b_{3}\right) \quad \lim \sup _{\xi \rightarrow 0^{+}} \frac{\operatorname{essinf}_{x \in j} F(x, \xi)}{|\xi|^{2}}=+\infty$

(b4) $\quad \liminf _{\xi \rightarrow 0^{+}} \frac{\operatorname{essinf}_{x \in J} F(x, \xi)}{|\xi|^{2}}>-\infty$.

Arguing as in [23, 24], let $u_{0}$ be an arbitrary solution of three solutions of problem 1.1. We shall show that the function $u_{0}$ cannot be trivial. Let us prove that

$$
\limsup _{\|u\| \rightarrow 0^{+}} \frac{\Psi_{\lambda, \mu}(u)}{\Phi(u)}=+\infty .
$$

In view of assumptions $\left(b_{3}\right)$ and $\left(b_{4}\right)$, we can consider a sequence $\left\{\xi_{n}\right\} \subset \mathbb{R}^{+}$converging to zero and two constants $\sigma, \kappa$ (with $\sigma>0$ ) such that

$$
\limsup _{n \rightarrow+\infty} \frac{\operatorname{essinf}_{x \in j} F\left(x, \xi_{n}\right)}{\left|\xi_{n}\right|^{2}}=+\infty
$$

and

$$
\text { ess } \inf _{x \in J} F(x, \xi) \geq \kappa|\xi|^{2}
$$

for every $\xi \in[0, \sigma]$. We consider a set $\omega \subset j$ of positive measure and a function $v \in X$ such that

$\left(\mathrm{k}_{1}\right) v(x) \in[0,1]$ for every $x \in[0,1]$

$\left(\mathrm{k}_{2}\right) v(x)=1$ for every $x \in \omega$

$\left(\mathrm{k}_{3}\right) v(x)=0$ for every $x \in[0,1] \backslash J$.

Hence, fix $M>0$ and consider a real positive number $\eta$ with

$$
M<\frac{\eta \operatorname{meas}(\omega)+\kappa \int_{J \backslash \omega}|v(x)|^{2} d x}{\frac{1+L}{2}\|v\|^{2}} .
$$

Then, there is $n_{0} \in \mathbb{N}$ such that $\xi_{n}<\sigma$ and

$$
\operatorname{essinf} F\left(x, \xi_{n}\right) \geq \eta\left|\xi_{n}\right|^{2}
$$


for every $n>n_{0}$. Now, from the second inequality of (3.1), for every $n>n_{0}$, by recalling the properties of the function $v$ (that is, $0<\xi_{n} v(x)<\sigma$ for $n$ large enough), we have

$$
\begin{aligned}
\frac{\Psi_{\lambda, \mu}\left(\xi_{n} v\right)}{\Phi\left(\xi_{n} v\right)} & \geq \frac{\int_{\omega} F\left(x, \xi_{n}\right) d x+\int_{J \backslash \omega} F\left(x, \xi_{n} v(x)\right) d x}{\Phi\left(\xi_{n} v\right)} \\
& >\frac{\eta \operatorname{meas}(\omega)+\kappa \int_{J \backslash \omega}|v(x)|^{2} d x}{\frac{1+L}{2}\|v\|^{2}}>M .
\end{aligned}
$$

Since $M$ could be arbitrarily large, it is achieved that

$$
\lim _{n \rightarrow \infty} \frac{\Psi_{\lambda, \mu}\left(\xi_{n} v\right)}{\Phi\left(\xi_{n} v\right)}=+\infty
$$

from which (3.4) clearly follows. Hence, there exists a sequence $\left\{\rho_{n}\right\} \subset X$ strongly converging to zero such that, for $n$ large enough, $\rho_{n} \in \Phi^{-1}\left(-\infty, r_{3}\right)$ and

$$
I_{\lambda, \mu}\left(\rho_{n}\right)=\Phi\left(\rho_{n}\right)-\lambda \Psi_{\lambda, \mu}\left(\rho_{n}\right)<0 .
$$

Since $u_{0}$ is a local minimum of the restriction of $I_{\lambda, \mu}$ to $\Phi^{-1}\left(-\infty, r_{3}\right)$, we obtain

$$
I_{\lambda, \mu}\left(u_{0}\right)<0
$$

which means $u_{0}$ is not trivial.

Now, we give the following direct conclusion of Theorem 3.1.

Theorem 3.3. Assume that there exist three positive constants $c_{1}, d$ and $c_{4}$ with $c_{1}<d<\frac{3}{8 \pi^{2}} \sqrt{\frac{3(1-L)}{2(1+L)}} c_{4}$, such that

$\left(\mathrm{B}_{1}\right) \quad \max \left\{\frac{\int_{0}^{1} F\left(x, c_{1}\right) d x}{c_{1}^{2}}, \frac{2 \int_{0}^{1} F\left(x, c_{4}\right) d x}{c_{4}^{2}}\right\}<\frac{\left(\frac{3}{2}\right)^{3}(1-L)}{\left(\frac{3}{2}\right)^{3}(1-L)+8 \pi^{4}(1+L)} \frac{\int_{\frac{3}{4}}^{1} F(x, d) d x}{d^{2}}$.

Then, for every

$$
\lambda \in] \frac{\left(\frac{1-L}{2}+4 \pi^{4}(1+L)\left(\frac{2}{3}\right)^{3}\right) d^{2}}{\int_{\frac{3}{4}}^{1} F(x, d) d x}, \frac{1-L}{2} \min \left\{\frac{c_{1}^{2}}{\int_{0}^{1} F\left(x, c_{1}\right) d x}, \frac{c_{4}^{2}}{2 \int_{0}^{1} F\left(x, c_{4}\right) d x}\right\},
$$

there exists $\delta_{\lambda, g}^{\prime}>0$, where

$$
\begin{array}{r}
\delta_{\lambda, g}^{\prime}:=\min \left\{\operatorname { m i n } \left\{\frac{(1-L) c_{1}^{2}-2 \lambda \int_{0}^{1} F\left(x, c_{1}\right) d x}{G^{c_{1}}}, \frac{(1-L) c_{4}^{2}-4 \lambda \int_{0}^{1} F\left(x, \frac{1}{\sqrt{2}} c_{4}\right) d x}{2 G^{\frac{1}{\sqrt{2}} c_{4}}},\right.\right. \\
\left.\frac{(1-L) c_{4}^{2}-4 \lambda \int_{0}^{1} F\left(x, c_{4}\right) d x}{2 G^{c_{4}}}\right\}, \\
\left.\frac{\left(\frac{2}{3}\right)^{3}(1+L) 4 \pi^{4} d^{2}-\lambda\left(\int_{\frac{3}{4}}^{1} F(x, d) d x-\int_{0}^{1} F\left(x, c_{1}\right) d x\right)}{G(d)-G^{c_{1}}}\right\}
\end{array}
$$

such that, for each $\mu \in\left[0, \delta_{\lambda, g}^{\prime}\left[\right.\right.$, problem (1.1) has at least three generalized solutions $u_{i}(i=1,2,3)$ such that $\left\|u_{i}\right\|_{\infty}<c_{4}, i=1,2,3$.

Proof. Choose $c_{2}=\frac{1}{\sqrt{2}} c_{4}$ and $c_{3}=c_{4}$. From $\left(B_{1}\right)$, we have

$$
\frac{\int_{0}^{1} F\left(x, c_{2}\right) d x}{c_{2}^{2}}=2 \frac{\int_{0}^{1} F\left(x, \frac{1}{\sqrt{2}} c_{4}\right) d x}{c_{4}^{2}} \leq 2 \frac{\int_{0}^{1} F\left(x, c_{4}\right) d x}{c_{4}^{2}}
$$




$$
<\frac{\left(\frac{3}{2}\right)^{3}(1-L)}{\left(\frac{3}{2}\right)^{3}(1-L)+8 \pi^{4}(1+L)} \frac{\int_{\frac{3}{4}}^{1} F(x, d) d x}{d^{2}}
$$

and

$$
\frac{\int_{0}^{1} F\left(x, c_{3}\right) d x}{\left(c_{3}^{2}-c_{2}^{2}\right)}=2 \frac{\int_{0}^{1} F\left(x, c_{4}\right) d x}{c_{4}^{2}}<\frac{\left(\frac{3}{2}\right)^{3}(1-L)}{\left(\frac{3}{2}\right)^{3}(1-L)+8 \pi^{4}(1+L)} \frac{\int_{\frac{3}{4}}^{1} F(x, d) d x}{d^{2}} .
$$

Moreover, since $c_{1}<d$ from $\left(B_{1}\right)$, we get

$$
\begin{aligned}
& \frac{\left(\frac{3}{2}\right)^{3}(1-L)}{8 \pi^{4}(1+L)} \frac{\int_{\frac{3}{4}}^{1} F(x, d) d x-\int_{0}^{1} F\left(x, c_{1}\right) d x}{d^{2}} \\
> & \frac{\left(\frac{3}{2}\right)^{3}(1-L)}{8 \pi^{4}(1+L)} \frac{\int_{\frac{3}{4}}^{1} F(x, d) d x}{d^{2}}-\frac{\left(\frac{3}{2}\right)^{3}(1-L)}{8 \pi^{4}(1+L)} \frac{\int_{0}^{1} F\left(x, c_{1}\right) d x}{c_{1}^{2}} \\
> & \frac{\left(\frac{3}{2}\right)^{3}(1-L)}{8 \pi^{4}(1+L)} \frac{\int_{\frac{3}{4}}^{1} F(x, d) d x}{d^{2}}-\frac{\left(\frac{3}{2}\right)^{6}(1-L)^{2}}{64 \pi^{8}(1+L)^{2}+8 \pi^{4}\left(\frac{3}{2}\right)^{3}(1+L)(1-L)} \frac{\int_{\frac{3}{4}}^{1} F(x, d) d x}{d^{2}} \\
= & \frac{\left(\frac{3}{2}\right)^{3}(1-L)}{\left(\frac{3}{2}\right)^{3}(1-L)+8 \pi^{4}(1+L)} \frac{\int_{\frac{3}{4}}^{1} F(x, d) d x}{d^{2}} .
\end{aligned}
$$

Hence, from $\left(B_{1}\right)$, (3.5) and (3.6), we observe that the assumption $\left(A_{1}\right)$ of Theorem 3.1 is fulfilled, and it follows the conclusion.

We want to point out the simple case of Theorem 3.3, in which the function $f$ has separated variables.

Theorem 3.4. Let $f_{1} \in L^{1}([0,1])$ and $f_{2} \in C(\mathbb{R})$ be two functions and $\tilde{F}(t)=\int_{0}^{1} f(\xi) d \xi$ for all $t \in \mathbb{R}$. Assume that there exist three positive constants $c_{1}, d$ and $c_{4}$ such that $c_{1}<d<\frac{3}{8 \pi^{2}} \sqrt{\frac{3(1-L)}{2(1+L)}} c_{4}$,

$$
\begin{aligned}
\left(\mathrm{B}_{2}\right) & \max \left\{\frac{\sup _{|t| \leq c_{1}} \tilde{F}(t) \int_{0}^{1} f_{1}(x) d x}{c_{1}^{2}}, \frac{2 \sup _{|t| \leq c_{4}} \tilde{F}(t) \int_{0}^{1} f_{1}(x) d x}{c_{4}^{2}}\right\} \\
< & \frac{\left(\frac{3}{2}\right)^{3}(1-L)}{\left(\frac{3}{2}\right)^{3}(1-L)+8 \pi^{4}(1+L)} \frac{\tilde{F}(d) \int_{\frac{3}{4}}^{1} f_{1}(x) d x}{d^{2}} .
\end{aligned}
$$

Then, for every

$$
\lambda \in\left(\frac{\left(\frac{1-L}{2}+4 \pi^{4}(1+L)\left(\frac{2}{3}\right)^{3}\right) d^{2}}{\tilde{F}(d) \int_{\frac{3}{4}}^{1} f_{1}(x) d x}, \frac{1-L}{2 \int_{0}^{1} f_{1}(x) d x} \min \left\{\frac{c_{1}^{2}}{\sup _{|t| \leq c_{1}} \tilde{F}(t)}, \frac{c_{4}^{2}}{2 \sup _{|t| \leq c_{4}} \tilde{F}(t)}\right\}\right),
$$

and for every non-negative continuous function $g: \mathbb{R} \rightarrow \mathbb{R}$, for each

$$
\begin{array}{r}
\mu \in\left[0, \min \left\{\operatorname { m i n } \left\{\frac{(1-L) c_{1}^{2}-2 \lambda \sup _{|t| \leq c_{1}} \tilde{F}(t) \int_{0}^{1} f_{1}(x) d x}{G^{c_{1}}},\right.\right.\right. \\
\left.\frac{(1-L) c_{4}^{2}-4 \lambda \sup _{|t| \leq \frac{1}{\sqrt{2}} c_{4}} \tilde{F}(t) \int_{0}^{1} f_{1}(x) d x}{2 G^{\frac{1}{\sqrt{2}} c_{4}}}, \frac{(1-L) c_{4}^{2}-4 \lambda \sup _{|t| \leq c_{4}} \tilde{F}(t) \int_{0}^{1} f_{1}(x) d x}{2 G^{c_{4}}}\right\}, \\
\left.\left.\frac{\left(\frac{1-L}{2}+4 \pi^{4}(1+L)\left(\frac{2}{3}\right)^{3}\right) d^{2}-\lambda\left(\tilde{F}(d) \int_{\frac{3}{4}}^{1} f_{1}(x) d x-\sup _{|t| \leq c_{1}} \tilde{F}(t) \int_{0}^{1} f_{1}(x) d x\right)}{G(d)-G^{c_{1}}}\right\}\right),
\end{array}
$$


the problem

$$
\left\{\begin{array}{l}
u^{(i v)}(x)=\lambda f_{1}(x) f_{2}(u)+h(u), \quad \text { in }[0,1] \\
u(0)=u^{\prime}(0)=u^{\prime \prime}(1)=0, \\
u^{\prime \prime \prime}(1)=\mu g(u(1))
\end{array}\right.
$$

has at least three generalized solutions $u_{i}(i=1,2,3)$ such that $\left\|u_{i}\right\|_{\infty}<c_{4}, i=1,2,3$.

Proof. Set $f(x, t)=f_{1}(x) f_{2}(t)$ for all $(x, t) \in[0,1] \times \mathbb{R}$. Since $F(x, t)=f_{1}(x) \tilde{F}(t)$, we obtain from $\left(B_{2}\right)$ $\left(B_{1}\right)$.

We give the following simple consequence of Theorem 3.3 when $\mu=0$.

Theorem 3.5. Let $f:[0,1] \times \mathbb{R} \rightarrow \mathbb{R}$ be a continuous function such that

(B) $\quad \lim _{t \rightarrow 0} \frac{f(x, t)}{|t|}=\lim _{|t| \rightarrow+\infty} \frac{f(x, t)}{|t|}=0 \quad \forall x \in[0,1]$.

Then, for every $\lambda>\bar{\lambda}$, where

$$
\bar{\lambda}=\left(\frac{1-L}{2}+4 \pi^{4}(1+L)\left(\frac{2}{3}\right)^{3}\right) \max \left\{\inf _{d>0} \frac{d^{2}}{\int_{\frac{3}{4}}^{1} F(x, d) d x}, \inf _{d<0} \frac{d^{2}}{\int_{\frac{3}{4}}^{1} F(x, d) d x}\right\}
$$

the problem (1.1), in the case $\mu=0$ possesses at least four distinct non-trivial solutions.

Proof. Arguing as in [25], we set

$$
f_{1}(x, t)= \begin{cases}f(x, t), & \text { if }(x, t) \in[0,1] \times[0,+\infty), \\ 0, & \text { otherwise, }\end{cases}
$$

and

$$
f_{2}(x, t)= \begin{cases}-f(x,-t), & \text { if }(x, t) \in[0,1] \times[0,+\infty), \\ 0, & \text { otherwise }\end{cases}
$$

and define $F_{1}(x, t)=\int_{0}^{t} f_{1}(x, \xi) d \xi$ for every $(x, t) \in[0,1] \times \mathbb{R}$. Fix $\lambda>\bar{\lambda}$, and let $d>0$ such that

$$
\lambda>\frac{\left(\frac{1-L}{2}+4 \pi^{4}(1+L)\left(\frac{2}{3}\right)^{3}\right) d^{2}}{\int_{\frac{3}{4}}^{1} F(x, d) d x} .
$$

From

$$
\lim _{t \rightarrow 0^{+}} \frac{f_{1}(x, t)}{t}=\lim _{t \rightarrow+\infty} \frac{f_{1}(x, t)}{t}=0
$$

there exist $c_{1}<d$ such that

$$
\frac{\int_{0}^{1} F_{1}\left(x, c_{1}\right) d x}{c_{1}^{2}}<\frac{1-L}{2 \lambda}
$$

and there is $c_{4}$ with $d<\frac{3}{8 \pi^{2}} \sqrt{\frac{3(1-L)}{2(1+L)}} c_{4}$ such that

$$
\frac{\int_{0}^{1} F_{1}\left(x, c_{4}\right) d x}{c_{4}^{2}}<\frac{1-L}{4 \lambda} .
$$

Then, $\left(B_{1}\right)$ in Theorem 3.3 is satisfied, and

$$
\lambda \in] \frac{\left(\frac{1-L}{2}+4 \pi^{4}(1+L)\left(\frac{2}{3}\right)^{3}\right) d^{2}}{\int_{\frac{3}{4}}^{1} F(x, d) d x}, \frac{1-L}{2} \min \left\{\frac{c_{1}^{2}}{\int_{0}^{1} F\left(x, c_{1}\right) d x}, \frac{c_{4}^{2}}{2 \int_{0}^{1} F\left(x, c_{4}\right) d x}\right\}[.
$$


Hence, the problem (1.1) with $\mu=0$ admits two positive solutions $u_{1}, u_{2}$. Next, arguing in the same way, from

$$
\lim _{t \rightarrow 0^{+}} \frac{f_{2}(x, t)}{t}=\lim _{t \rightarrow+\infty} \frac{f_{2}(x, t)}{t}=0
$$

we ensure the existence of two positive solutions $u_{3}, u_{4}$ for the problem (1.1) with $\mu=0$. Clearly, $-u_{3},-u_{4}$ are negative solutions of the problem (1.1) with $\mu=0$ and the conclusion is achieved.

Remark 3.6. We obviously observe that in Theorem 3.5 no symmetric condition on $f$ is assumed. In the event that, $f$ is an odd continuous non-zero function such that $f(x, t)>0$ for all $(x, t) \in[0,1] \times(0,+\infty)$, $\left(B_{3}\right)$ can be replaced by

(B 4$) \quad \lim _{t \rightarrow 0^{+}} \frac{f(x, t)}{t}=\lim _{t \rightarrow+\infty} \frac{f(x, t)}{t}=0, \quad \forall x \in[0,1]$,

ensuring the existence of at least four distinct non-trivial solutions the problem (1.1) in the case $\mu=0$ for every $\lambda>\lambda^{*}$, where

$$
\lambda^{*}=\inf _{d>0} \frac{\left(\frac{1-L}{2}+4 \pi^{4}(1+L)\left(\frac{2}{3}\right)^{3}\right) d^{2}}{\int_{\frac{3}{4}}^{1} F(x, d) d x} .
$$

Remark 3.7. When $f$ does not depend on $x$, hypotheses $\left(B_{1}\right)$ become to the following simpler form:

$$
\begin{aligned}
\left(\mathrm{B}_{1}^{\prime}\right) \max \left\{\frac{F\left(c_{1}\right)}{c_{1}^{2}}, \frac{2 F\left(c_{4}\right)}{c_{4}^{2}}\right\}<\frac{1}{4} \frac{\left(\frac{3}{2}\right)^{3}(1-L)}{\left(\frac{3}{2}\right)^{3}(1-L)+8 \pi^{4}(1+L)} \frac{F(d)}{d^{2}}, \\
\lambda \in\left(\frac{\left(\frac{3}{2}\right)^{3}(1-L)+8 \pi^{4}(1+L)}{\left(\frac{3}{2}\right)^{3}} \frac{2 d^{2}}{F(d)}, \frac{1-L}{2} \min \left\{\frac{c_{1}^{2}}{F\left(c_{1}\right)}, \frac{c_{4}^{2}}{2 F\left(c_{4}\right)}\right\}\right),
\end{aligned}
$$

and

$$
\begin{aligned}
& \delta_{\lambda, g}^{\prime \prime}:=\min \left\{\operatorname { m i n } \left\{\frac{(1-L) c_{1}^{2}-2 \lambda F\left(c_{1}\right)}{G^{c_{1}}}, \frac{(1-L) c_{4}^{2}-4 \lambda F\left(\frac{1}{\sqrt{2}} c_{4}\right)}{2 G^{\frac{1}{\sqrt{2}} c_{4}}},\right.\right. \\
& \left.\left.\frac{(1-L) c_{4}^{2}-4 \lambda F\left(c_{4}\right)}{2 G^{c_{4}}}\right\}, \frac{\left(\frac{2}{3}\right)^{3}(1+L) 4 \pi^{4} d^{2}-\lambda\left(\frac{1}{4} F(d)-F\left(c_{1}\right)\right)}{G(d)-G^{c_{1}}}\right\} .
\end{aligned}
$$

Theorem 3.8. Let $f, g: \mathbb{R} \rightarrow[0,+\infty[$ be two continuous functions. Assume that there exist three positive constants $c_{1}, \bar{d}$ and $c_{4}$ with $c_{1}<2 \bar{d}<\frac{3}{8 \pi^{2}} \sqrt{\frac{3(1-L)}{2(1+L)}} c_{4}$ such that

$\left(\mathrm{B}_{5}\right) f(t)<\frac{1-L}{2} c_{1}$ for all $t \in\left[0, c_{1}\right]$;

(B 6$) f(t)>\left(\frac{27(1-L)+64 \pi^{4}(1+L)}{\left(\frac{3}{2}\right)^{3}}\right) \bar{d}$ for all $t \in[\bar{d}, 2 \bar{d}]$;

(B $\left.\mathrm{B}_{7}\right) f(t)<\frac{1-L}{4} c_{4}$ for all $t \in\left[0, c_{4}\right]$.

Then, the problem

$$
\left\{\begin{array}{l}
u^{(i v)}(x)=f(u)+h(u), \quad \text { in }[0,1], \\
u(0)=u^{\prime}(0)=u^{\prime \prime}(1)=0, \\
u^{\prime \prime \prime}(1)=0,
\end{array}\right.
$$

for the case $\mu=0$, has at least three classical solutions $u_{i}(i=1,2,3)$ such that $\left\|u_{i}\right\|_{\infty}<c_{4}, i=1,2,3$.

Proof. All the assumptions of Theorem 3.3 are satisfied by choosing $d=2 \bar{d}$ and $F(t)=\int_{0}^{t} f(\xi) d \xi$ for all $t \in \mathbb{R}$. From our assumptions, we obtain

$$
\int_{0}^{c_{1}} f(t) d t<\frac{1-L}{2} c_{1}^{2}
$$




$$
\begin{gathered}
\int_{0}^{d} f(t) d t \geq \int_{\bar{d}}^{2 \bar{d}} f(t) d t>\left(\frac{\left(27(1-L)+64 \pi^{4}(1+L)\right.}{\left(\frac{3}{2}\right)^{3}}\right) \vec{d}^{2} \\
=\left(\frac{2\left(\frac{3}{2}\right)^{3}(1-L)+16 \pi^{4}(1+L)}{\left(\frac{3}{2}\right)^{3}}\right) d^{2}, \\
\int_{0}^{c_{4}} f(t) d t<\frac{1-L}{4} c_{4}^{2} .
\end{gathered}
$$

That is, $\frac{F\left(c_{1}\right)}{c_{1}^{2}}<\frac{1-L}{2}$,

$$
\frac{F(d)}{d^{2}}>\frac{2\left(\frac{3}{2}\right)^{3}(1-L)+16 \pi^{4}(1+L)}{\left(\frac{3}{2}\right)^{3}}, \frac{F\left(c_{4}\right)}{c_{4}^{2}}<\frac{1-L}{4} .
$$

Therefor, the conditions in Remark 3.7 are verified and

$$
1 \in] \frac{2\left(\frac{3}{2}\right)^{3}(1-L)+16 \pi^{4}(1+L)}{\left(\frac{3}{2}\right)^{3}} \frac{d^{2}}{F(d)}, \frac{1-L}{2} \min \left\{\frac{c_{1}^{2}}{F\left(c_{1}\right)}, \frac{1}{2} \frac{c_{4}^{2}}{F\left(c_{4}\right)}\right\}[
$$

Hence, taking Remark 3.7 again into account, Theorem 3.3 ensures the conclusion.

We now present the following example to illustrate Theorem 3.1.

Example 3.9. Consider the problem

$$
\left\{\begin{array}{l}
u^{(i v)}(x)=\lambda f(x, u(x))+\sin \left(\frac{u}{2}\right), \quad \text { in }[0,1] \\
u(0)=u^{\prime}(0)=u^{\prime \prime}(1)=0 \\
u^{\prime \prime \prime}(1)=\mu g(u(1))
\end{array}\right.
$$

For all $(x, t) \in[0,1] \times \mathbb{R}$, we put $f(x, t)=x f(t)$, where

$$
f(t)= \begin{cases}4 t^{3}, & \text { if } t \leq 1 \\ 4 t, & \text { if } 1<t \leq 2 \\ 8, & \text { if } 2<t \leq 3 \\ \frac{72}{t^{2}}, & \text { if } t>3\end{cases}
$$

By the expression of $f$, we have

$$
F(t)= \begin{cases}t^{4}, & \text { if } t \leq 1 \\ 2 t^{2}-1, & \text { if } 1<t \leq 2 \\ 8 t-9, & \text { if } 2<t \leq 3 \\ \frac{117}{3}-\frac{72}{t}, & \text { if } t>3\end{cases}
$$


By choosing $d=2, c_{1}=10^{-4}, c_{2}=10^{4}$ and $c_{3}=\sqrt{2} \times 10^{4}$, we see that

$$
\begin{aligned}
& \max \left\{\frac{\int_{0}^{1} F\left(x, c_{1}\right) d x}{c_{1}^{2}}, \frac{\int_{0}^{1} F\left(x, c_{2}\right) d x}{c_{2}^{2}}, \frac{\int_{0}^{1} F\left(x, c_{3}\right) d x}{c_{3}^{2}-c_{2}^{2}}\right\} \\
& =\frac{1}{2 \times 10^{8}} \times \frac{117 \times 10^{4}-216}{3 \times 10^{4}} \\
& <\frac{9}{512 \pi^{4}}\left(\frac{49}{16}-10^{-16}\right) \\
& =\left(\frac{3}{2}\right)^{3} \frac{1-L}{1+L} \frac{\int_{\frac{3}{4}}^{1} F(x, d) d x-\int_{0}^{1} F\left(x, c_{1}\right) d x}{8 \pi^{4} d^{2}},
\end{aligned}
$$

which means the assumption $(A 1)$ is satisfied. It is easy to see that other assumptions of Theorem 3.1 are also fulfilled. Therefore, it follows that, for every

$$
\lambda \in\left(\frac{8192 \pi^{4}}{9\left(49-16 \times 10^{-16}\right)}, \frac{6 \times 10^{12}}{117 \times 10^{4}-216}\right)
$$

and for every non-negative continuous function $g: \mathbb{R} \rightarrow \mathbb{R}$, there exists $\hat{\delta}>0$ such that, for each $\mu \in$ $[0, \hat{\delta})$, problem (1.1) has at least three classical solutions $u_{1}, u_{2}$ and $u_{3}$ such that $\left\|u_{i}(x)\right\|_{\infty}<\sqrt{2} \times 10^{4}, i=$ $1,2,3$.

\section{REFERENCES}

[1] G.A. Afrouzi, S. Heidarkhani, D. O’Regan, Existence of three solutions for a doubly eigenvalue fourth-order boundary value problem, Taiwanese J. Math. 15 (2011), 201-210.

[2] D. Averna, G. Bonanno, A mountain pass theorem for a suitable class of functions, Rocky Mountain J. Math. 39 (2009), 707-727.

[3] Z. Bai, H. Wang, On positive solutions of some nonlinear fourth-order beam equations, J. Math. Anal. Appl. 270 (2002), 357-368.

[4] G. Bonanno, A. Chinnì, S.A. Tersian, Existence results for a two point boundary value problem involving a fourth-order equation, Electron. J. Qual. Theory Differ. Equ. 2015 (2015), Article ID 33.

[5] G. Bonanno, B. Di Bella, A boundary value problem for fourth-order elastic beam equations, J. Math. Anal. Appl. 343 (2008), 1166-1176.

[6] G. Bonanno, B. Di Bella, Infinitely many solutions for a fourth-order elastic beam equation, Nonlinear Differ. Equ. Appl. 18 (2011), 357-368.

[7] G. Bonanno, B. Di Bella, D. O’Regan, Non-trivial solutions for nonlinear fourth-order elastic beam equations, Comput. Math. Appl. 62 (2011), 1862-1869.

[8] G. Bonanno, A. Sciammetta, An existence result of one non-trivial solution for two point boundary value problems, Bull. Aust. Math. Soc. 84 (2011), 288-299.

[9] A. Cabada, J.A. Cid, L. Sanchez, Positivity and lower and upper solutions for fourth order boundary value problems, Nonlinear Anal. 67 (2007), 1599-1612.

[10] A. Cabada, S.A. Tersian, Multiplicity of solutions of two point boundary value problem for a fourth-order eequation, Appl. Math. Comput. 219 (2013), 5261-5267.

[11] M.R. Grossinho, S.A. Tersian, The dual variational principle and equilibria for a beam resting on a discontinuous nonlinear elastic foundation, Nonlinear Anal. 41 (2000), 417-431.

[12] A. Hadjian, M. Ramezani, Existence of infinitely many solutions for fourth-order equations depending on two parameters, Electron. J. Differential Equations, 2017 (2017), Article ID 117.

[13] S. Heidarkhani, M. Ferrara, A. Salari, M. Azimbagirad, A variational approach to perturbed elastic beam problems with nonlinear boundary conditions, Math. Reports 18 (2016), 573-589. 
[14] T.F. Ma, Positive solutions for a beam equation on a nonlinear elastic foundation, Math. Comput. Modelling 39 (2004), $1195-1201$

[15] T.F. Ma, J.d. Silva, Iterative solutions for a beam equation with nonlinear boundary conditions of third order, Appl. Math. Comput. 159 (2004), 11-18.

[16] Y. Song, A nonlinear boundary value problem for fourth-order elastic beam equations, Bound. Value Probl. 2014 (2014), 1-11.

[17] L. Yang, H. Chen, X. Yang, The multiplicity of solutions for fourth-order equations generated from a boundary condition, Appl. Math. Lett. 24 (2011), 1599-1603.

[18] G. Bonanno, P. Candito, Non-differentiable functionals and applications to elliptic problems with discontinuous nonlinearities, J. Differential Equations 244 (2008), 3031-3059.

[19] S. Heidarkhani, G.A. Afrouzi, S. Moradi, G. Caristi, A variational approach for solving $p(x)$-biharmonic equations with Navier boundary conditions, Electron. J. Differential Equations, 2017 (2017), Article ID 25.

[20] S. Heidarkhani, A.L.A. De Araujo, G.A. Afrouzi, S. Moradi, Multiple solutions for Kirchhoff-type problems with variable exponent and nonhomogeneous Neumann conditions, Math. Nachr. 291 (2018) 326-342.

[21] L. Kong, Existence of solutions to boundary value problems arising from the fractional advection dispersion equation, Electron. J. Differential Equations, 2013 (2013), Article ID 106.

[22] E. Zeidler, Nonlinear functional analysis and its applications, Vol. II/B, Springer, Berlin, Heidelberg, New York, 1985.

[23] G. Bonanno, Relations between the mountain pass theorem and local minima, Adv. Nonlinear Anal. 1 (2012), $205-220$.

[24] M. Galewski, G. Molica Bisci, Existence results for one-dimensional fractional equations, Math. Meth. Appl. Sci. 39 (2016), 1480-1492.

[25] G. Bonanno, P. Candito, Nonlinear difference equations investigated via critical point methods, Nonlinear Anal. 70 (2009), 3180-3186. 